\title{
La Visión del Informe Sandford en apoyo a Esquipulas II para fundamentar la cultura de paz en Centroamérica
}

${ }^{1}$ Juan B. Arrien

\section{RESUMEN}

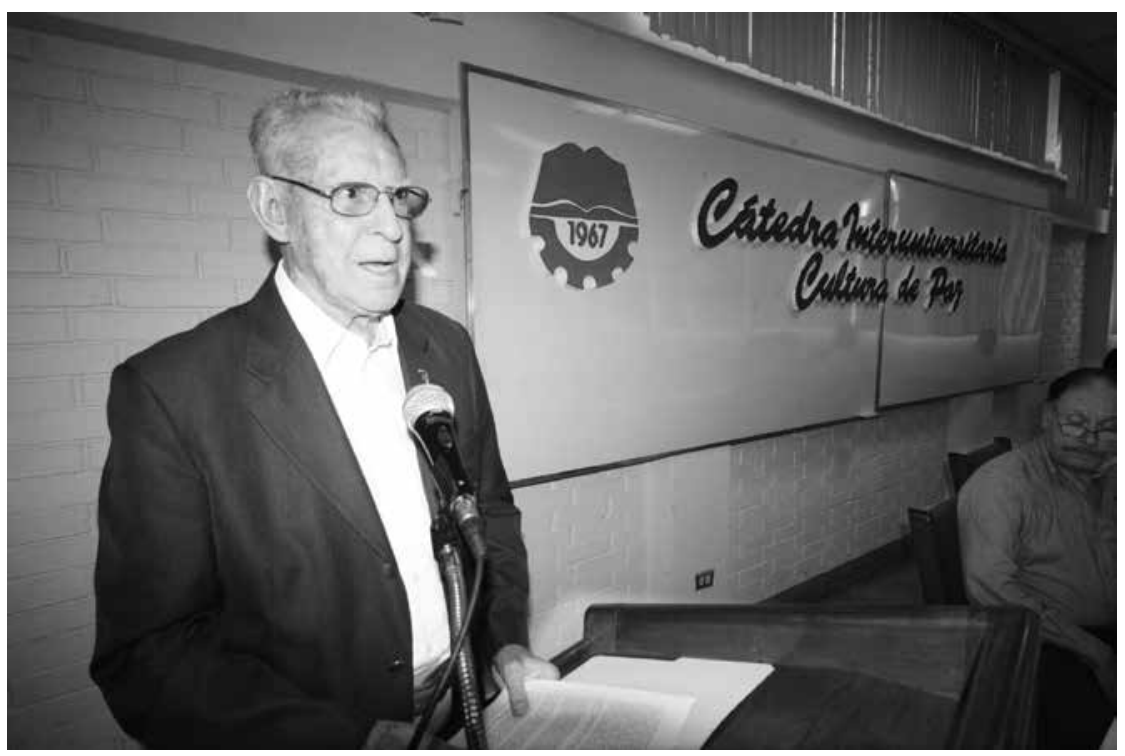

Foto: Edward Dávila

\section{El Informe Sandford}

L a guerra parió la paz y la paz comenzó a ser cultura en Nicaragua.

A finales del año 1989, estuve involucrado en la preparación de la Conferencia Mundial de Educación, Jomtien, Thailandia, marzo de 1990.

La Oficina Subregional de educación de la UNESCO para Centroamérica y Panamá con sede en San José, Costa Rica, me encomendó preparar un estudio ensayo que proporcionara una visión y análisis ajustado a la realidad de la educación básica en la región.

La UNESCO, lo publicó con el título “La Educación Básica en Centroamérica y Panamá" frente al desafío de universalizarla para el año 2000 (análisis histórico)".
Juan Bautista Arríen, filósofo y educador, rescata, actualiza y destaca en su valor histórico el Informe Sandford para Centroamérica de 1989, el cual ubica a la pobreza como la raíz profunda de los conflictos violentos que agotaron la región en la década de los 80 .

El Informe Sandford identifica la paz, la democracia y desarrollo como el fundamento para una paz duradera y sostenible, coincidiendo con la UNESCO que ve en este triángulo interactivo la base de la cultura de paz.

Palabras clave: Informe, conflicto, paz, democracia, desarrollo.

\section{ABSTRACT}

The Sandford Report identifies peace, democracy and development as the foundation for a lasting and sustainable peace, coinciding with UNESCO which sees in this interactive triangle the basis for a culture of peace.

Key words: report, conflict, peace, democracy, development.

1 Secretario Permanente Comisión Nacional de UNESCO (Nicaragua). 


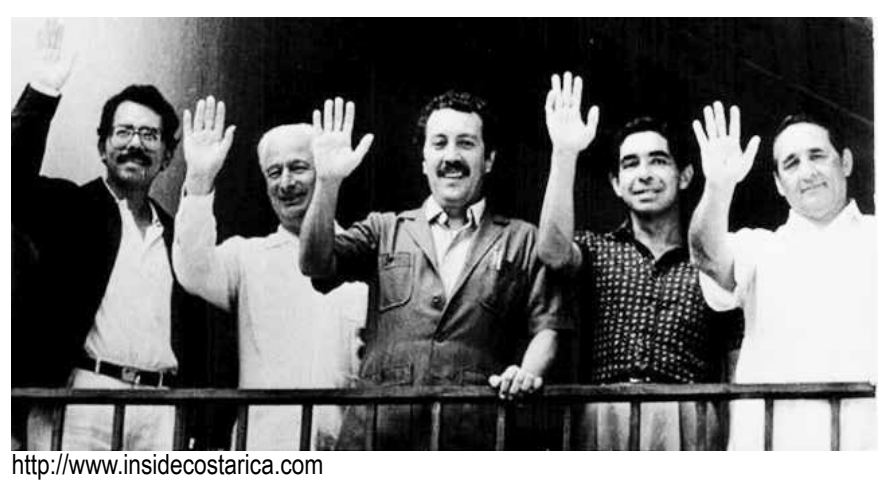

Envuelto en dicho quehacer cayó en mis manos una fotocopia del Informe Sandford producto del trabajo de la Comisión Internacional para la recuperación y desarrollo de Centroamérica, antes que se publicara en Madrid 1989 con el título "Pobreza, Conflicto y Esperanza: Un momento crítico para Centroamérica". Sobra decir que me puso en bandeja de plata el contexto a fondo de la realidad centroamericana en la cual la visión de la educación presentaba un sentido de profundidad y de relaciones inseparables con los distintos factores que influyen en ella y que en cierto sentido la definen y determinan. En otras palabras, el Informe guió mi trabajo para engrosar los estudios que estábamos preparando los expertos a quienes el Banco Mundial, el PNUD, la UNESCO y UNICEF encomendaron el material que fundamentaría las deliberaciones propuestas y resultados de la Conferencia Mundial sobre Educación para Todos.

El momento crítico al que alude el Informe Sandford, producto del trabajo de 147 personas de distintas regiones del mundo, especialistas en las distintas disciplinas que fundamentan y sostienen la paz, la democracia y el desarrollo, tenía sus raíces más hondas en la pobreza, con todo lo que invade y genera, es decir, el conflicto, la guerra destructora e inhumana y en cuyas entrañas se movía con celeridad la esperanza, la paz.

El Informe persiguió en el fondo hacer efectivos tres componentes inseparables, la paz, la democracia y el desarrollo componentes que nunca han estado juntos y plenos en Centroamérica y que por ese tiempo tenían una connotación inversa, negativa.

La visión del Informe Sandford, su análisis y su propuesta para hacer realidad en una unidad inseparable, coincide con la opinión del Director General de la UNESCO, profesor Federico Mayor Zaragoza cuando en su declaración "El Derecho Humano a la Paz" 1997 escribió "Paz, desarrollo y democracia forman un triángulo interactivo. Los tres se requieren mutuamente". El Informe Sandford integra el triángulo con los tres lados que lo conforman aunque en una secuencia propia, histórica: la paz, superación de la guerra, la democracia, participación de todos en el accionar del país, el desarrollo equitativo en beneficio de todos.

Esa paz era el primer paso la que se convertiría en cultura cuando la completaran la democracia y el desarrollo. Es decir, "la paz duradera premisa y requisito para el ejercicio de todos los derechos y deberes humanos; no la paz del silencio, no de los hombres y mujeres silenciosos, silenciados. La paz de la libertad y por tanto de las leyes justas, de la alegría, de la igualdad, de la solidaridad donde todos los ciudadanos cuentan, conviven, comparten", según Federico Mayor (o.c.).

El Informe Sandford señaló la ruta de la cultura de paz con gran precisión pero una vez que esta se liberara del dominio de la guerra. Ahí se hace presente Esquipulas II.

Resulta muy interesante volver a entender y vivir el proceso de paz en Centroamérica para después comprender los componentes de una cultura de paz tal como lo planteó el Informe Sandford y lo concretaron los presidentes centroamericanos y sus pueblos en el conocido proceso de paz definidos en Esquipulas II.

A la par que se logró la paz contra la guerra se asientan los fundamentos de la Cultura de Paz, con la democracia y el desarrollo que la sustentan. Para ello conviene tener presente el contexto centroamericano de los ochentas.

\section{El contexto Centroamericano de los años 80}

Es difícil afirmar categóricamente, pero al menos existen razones para hacerlo, que Centroamérica viene a ser una síntesis de lo que ha sido en realidad la década de los 80 para muchos países del tercer mundo.

Esta década de los 80 ha sido catalogada por muchos como la década perdida, la década del retroceso. En verdad Centroamérica se convirtió en sinónimo de derramamiento de sangre, de injusticia y de pobreza. La violencia ha arrancado de sus hogares y pueblos a casi tres 
millones de centroamericanos (desplazados, refugiados, exiliados, etc.) es decir al 15\% de la población, dejando a la mayoría sin trabajo, sin ingresos suficientes y con precarios servicios de salud y educación; alrededor de 170.000 centroamericanos murieran en las guerras y en la violencia durante los últimos diez años.

Esta situación impulsó a los presidentes centroamericanos asumir como propia la tarea de buscar una solución a la crisis, sustituyendo al Grupo de Contadora que con buena voluntad pero desde el exterior quería dar una solución a Centroamérica. Esta solución la asume la misma Centroamérica a través de sus gobernantes y a partir de ésta la voluntad de paz, de democracia, de desarrollo se fue fortaleciendo, y los planteamientos y las decisiones se hicieron cada vez más cercanos a la paz, cada vez más integrales, por cuanto empezó a concebirse que la paz, la democracia y el desarrollo son lógicamente inseparables.

Se trata de la premisa fundamental y es que la paz duradera, la democracia auténtica y el desarrollo equitativo son, inseparables, ninguno de esos tres objetivos es suficiente por él solo, cada uno es igualmente necesario para la consecución de los demás y es necesario promoverlos simultáneamente. Sin paz no puede haber desarrollo, sin un desarrollo equitativo la democracia verdadera no es posible y sin democracia no habrá paz duradera. Es importante tener presente esta nueva concepción emanada de la voluntad de los presidentes centroamericanos, expresada a partir de Esquipulas I (Guatemala, mayo 86), pasando por Esquipulas II

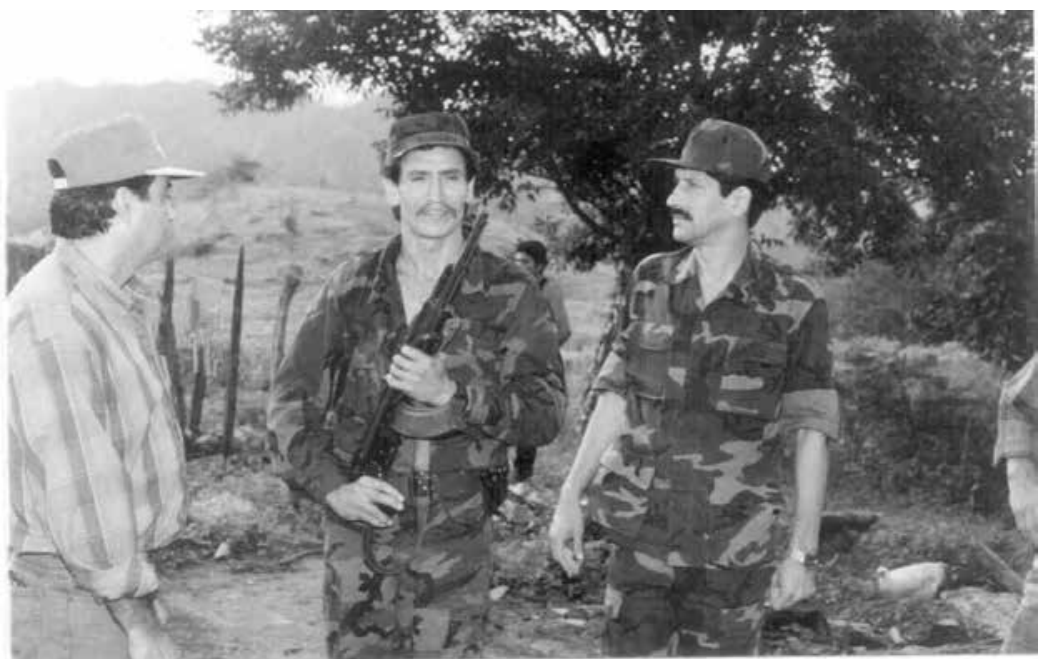

(agosto 87), siguiendo por Alajuela (Costa Rica, febrero 88), continuando en Costa del Sol (El Salvador, agosto 88), avanzando por Tela (Honduras, junio 89), y en diciembre 89 en San Isidro de Coronado, Costa Rica. De tal manera que si bien el plan para lograr una paz firme y duradera viene a constituirse en título de todos estos esfuerzos, la verdad es que esa paz firme y duradera está intrínseca e inseparablemente unida a la democracia y al desarrollo, tal como lo expresaron y asumieron los presidentes centroamericanos en sus gestiones para proporcionar a Centroamérica un nuevo rostro humano y social.

\section{Esquipulas II}

Sin embargo la célula madre de este ansiado proceso fue Esquipulas II (agosto 1987) porque además de luchar por alcanzar la paz, en su espíritu, contenido y direccionalidad, están los componentes esenciales de la Cultura de Paz.

Esquipulas II sentó las bases, señaló el camino y definió los componentes de una Cultura de Paz cuya consolidación se encuentra todavía en deuda en la actualidad pese a haber enterrado definitivamente el imperio de las armas tal como lo confirman los acuerdos de Sapóa (marzo 1988) y la entrega de las armas por el Comandante Franklin de la contra a la Presidenta Violeta de Chamorro en San Pedro de Lóvago, Nicaragua (1990). Los acuerdos de paz El Salvador (1992) y los de Guatemala (1996).

Hemos avanzado pero el ritmo es lento y de no actuar con inteligencia, compromiso y acción podríamos volver a situaciones algo cercanas a las que se apoderaron de la negación de la paz auténtica, la democracia verdadera y el desarrollo equitativo en la década del 80.

Realmente emociona releer los acuerdos de Esquipulas II, cuando revivimos las vivencias y el recuerdo de la caótica situación que presentaba Centroamérica durante varios años de la década del 80 .

En dichos acuerdos se activan elementos esenciales que tienen palpable vigencia cuando nos esforzamos por construir una Cultura de Paz en Nicaragua.

Echemos una mirada con detenimiento y osadía: 


\section{El diálogo}

En el preámbulo se acuerda: "hacer prevalecer el diálogo sobre la violencia y la razón sobre los rencores". No cabe duda que el diálogo es un permanente acompañante de la Cultura de Paz porque se basa en la igualdad esencial de todas las personas y por ende el respeto a sus derechos, ideas y opiniones en la búsqueda del bien común. Este diálogo proactivo no siempre es el invitado en la vida política, económica y social del país para el logro de consensos de un proyecto de nación, cuando existen divisiones profundas alimentadas por intereses que a la postre no abonan al bienestar de la población y hacen muy difícil el funcionamiento adecuado de una verdadera ciudadanía en Nicaragua. Todavía tienen vida varias formas de rencores que impiden la convivencia ciudadana y la materializan en corrupción, falta de veracidad y violencia multiforme. Dialogan los intereses pero no el bienestar de la población, sustento de la Cultura de Paz.

Respecto al diálogo Esquipulas II sigue presente cuando propugna:

“Realizar urgentemente en aquellos casos donde se han producido profundas divisiones dentro de la sociedad, acciones de reconciliación nacional que permitan la participación popular, con garantía plena, en auténticos procesos políticos de carácter democrático, sobre bases de justicia, libertad y democracia y, para tal efecto, crear los mecanismos que permitan, de acuerdo con la ley, el diálogo con los grupos opositores". Sin duda que el mensaje trasmite sonidos de Cultura de Paz que muchos oídos en Nicaragua no quieren escuchar.

\section{La democracia}

Respecto a la democratización que se concreta en una verdadera democracia, Esquipulas II va directo al grano.

“Los gobiernos se comprometen a impulsar un auténtico proceso democrático pluralista y participativo que implique la promoción de la justicia social, el respeto de los Derechos Humanos, la soberanía, la integridad territorial de los Estados y el derecho de todas las naciones a determinar libremente y sin injerencias externas de

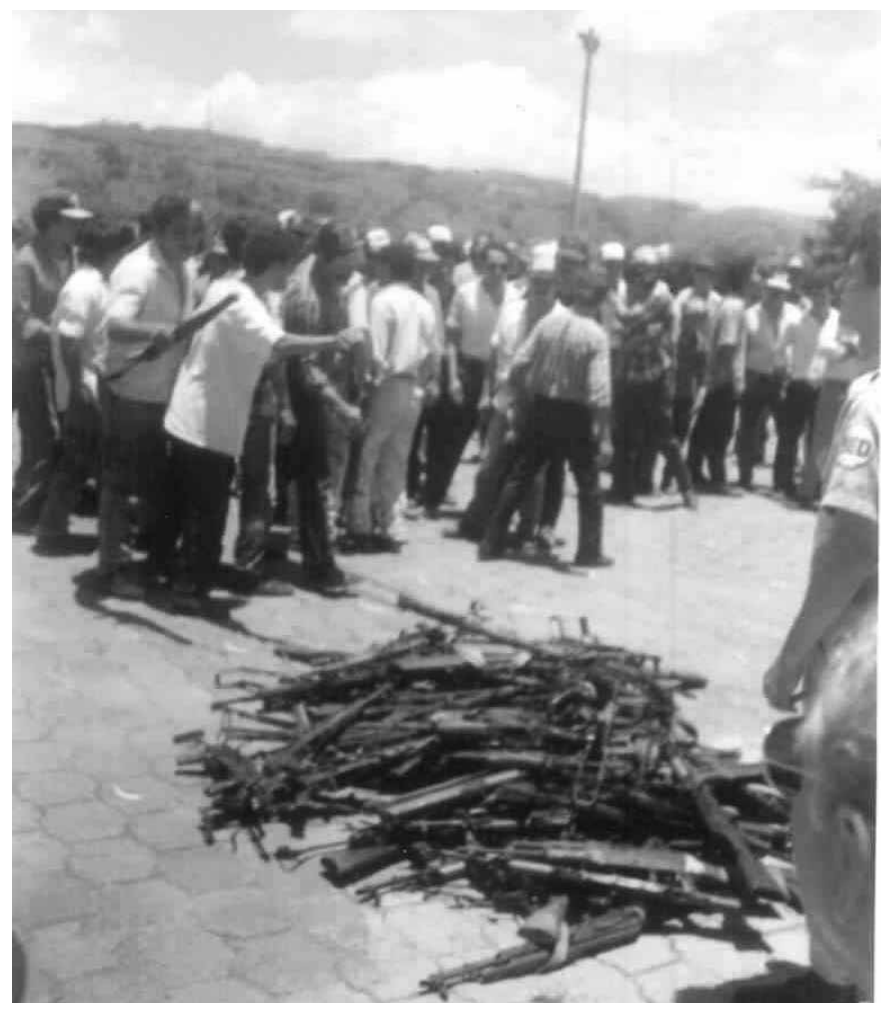


permitan acelerar el desarrollo, para alcanzar sociedades más igualitarias y libres de la miseria. La consolidación de la democracia implica la creación de un sistema de bienestar y justicia económica y social. Para lograr estos objetivos los gobiernos gestionarán conjuntamente un apoyo económico extraordinario de la Comunidad Internacional".

El desarrollo inclusivo, económico, humano y social queda todavía lejos en Nicaragua cuando lo medimos desde la equidad y sus distintas formas de exclusión para construir una Cultura de Paz.

\section{El aporte del Informe Sandford para materializar la esencia de Esquipulas II.}

En este contexto el Informe Sandford jugó un papel clave en apoyo a la misión plena de Esquipulas II para que Centroamérica se levantara de su postración humana, social, económica y emocional activando los elementos que le dieran nueva vida a través de los tres componentes inseparables, que se necesitan mutuamente, la paz, la democracia, el desarrollo, mismos que con sus respectivos elementos constituyen los verdaderos componentes de una Cultura de Paz en nuestros días.

Efectivamente, la Comisión Internacional para la recuperación y desarrollo de Centroamérica, cuyo trabajo como se dijo, es conocido como Informe Sandford, después de hacer un análisis completo de la situación de cada uno de los países de Centroamérica y tener una visión global de la región y sumar su voz a la de 147 expertos internacionales de diversas disciplinas para emitir su penetrante propuesta a fin de fomentar el proceso pacificador, robustecer y estabilizar las instituciones democráticas, ampliar la participación propuesta y proporcionar una base sólida para el desarrollo socioeconómico.

Los problemas están profundamente arraigados en la pobreza endémica y en la injusticia que durante mucho tiempo han plagado la región colocándola en un círculo vicioso muy difícil de revertir.

Para ello la Comisión define la premisa fundamental y es que la paz duradera, la democracia auténtica y el desarrollo equitativo son inseparables.
Ninguno de esos elementos es suficiente por sí solo, cada uno es igualmente necesario para la consecución de los demás. Centroamérica se encontraba en un momento crucial de su historia, que requería la adopción de medidas rápidas y decisivas pero con un plan de acción inmediata y visión de largo plazo.

En este sentido el plan de acción inmediata con mucho humanismo, ternura y claridad; recomienda:

- Prestar especial atención a los refugiados y desplazados , así como a las personas que vivan en la indigencia en las comunidades en las cuales se hayan reasentado o reubicado.

- Incluir medidas especiales para los niños menores de cinco años, las mujeres embarazadas y lactantes, y los ancianos, y;

- Fomentar la creación de fuentes de trabajo, teniendo en cuenta los aspectos de la seguridad alimentaria, salud y nutrición, educación básica, abastecimiento de agua potable y servicios de saneamiento, vivienda temporaria, infraestructura y derechos humanos.

Más a largo plazo en la perspectiva del desarrollo sostenido el informe afirma que el primer paso es poner fin al enfrentamiento armado y restablecer un clima propicio para la inversión. Sólo mediante el crecimiento económico, Centroamérica podrá generar los recursos necesarios para aliviar la pobreza y superar el nivel de vida de toda la población.

La Comisión concluye entre otros factores muy precisos de orden económico y de gestión pública:

- La inversión y la productividad han mermado considerablemente; por consiguiente, se necesitan políticas encaminadas a desarrollar el potencial humano desaprovechado y conservar los frágiles recursos naturales de la región.

- La mejora de las condiciones socioeconómicas de la región debe basarse en el crecimiento económico, la creación de las fuentes de trabajo y una distribución equitativa de los beneficios del crecimiento.

- La mejor base para una estrategia de desarrollo eficaz consiste en el desarrollo de los recursos humanos, el aumento de la productividad, la promoción de las 
exportaciones, la integración regional y la seguridad alimentaria.

- Se reformen las escuelas de la región asignando prioridad a la educación primaria.

- Se restauren y reformen los sistemas de salud en Centroamérica, pilares y soportes del desarrollo humano.

Respecto a la democracia. En Centroamérica la ausencia de democracia ha sido una de las causas principales de conflicto, cuando es la democracia la que fomenta el desarrollo equitativo y promueve la paz.

Durante muchos años dominaban en Centroamérica (excepto Costa Rica) los gobiernos militares algunos de ellos fueron derrocados y otros tuvieron que ceder a la presión de la población lo que hizo que se avanzara hacia la participación democrática, los partidos políticos intensificaron sus actividades, en las elecciones aumenta el número de votantes y tomaran mucha fuerza las organizaciones de base y de la sociedad.

Esto anunciaba la necesidad de promover más activamente la democracia y la participación popular en Centroamérica dando vida a algunos organismos e instituciones que las impulsaran y le proporcionaran mayor ritmo y consistencia.

A este respecto la Comisión recomienda por ejemplo, la creación de un Consejo de Cultura y Educación con el fin de incentivar el espíritu democrático y el desarrollo de los recursos humanos propuesta que se hizo realidad más adelante aunque sin demasiada fuerza en la Coordinadora Educativa y Cultural de Centroamérica (CECC).

La Comisión también recomendó el establecimiento de un Consejo Económico y Social que actuara como órgano consultivo económico y social de la Sociedad Civil a fin de fomentar la democracia.

Estas semillas cayeron en tierra fértil lo que posibilitó contar ahora por ejemplo con el Sistema de Integración Centroamericana (SICA) aunque algo diferente y quizás desvirtuado del concebido por el Informe Sandford.
No cabe duda que el Informe Sandford visualizó con mucho acierto el futuro relativamente cercano.

Por otra parte el Informe Sandford siempre tiene presente la realidad regional y la visualiza en términos de su propia e interna cooperación.

A este respecto la Comisión visualizó plataformas conjuntas de los gobiernos centroamericanos con el fin de proporcionar bases sólidas para la paz, la democracia y el desarrollo, tales como:

- Mejorar la coordinación de las políticas fiscales y monetarias, así como de las tasas de interés y los tipos de cambio.

- Establecer un Parlamento Centroamericano y promover su afianzamiento a fin de abrir nuevos canales para el diálogo político regional, y;

- Crear una Corte Interamericana de Justicia con objeto de facilitar la solución de controversias y encontrar soluciones justas en los casos en que no sea posible alcanzarlas empleando otros mecanismos institucionales.

En tanto Comisión Internacional para la recuperación y desarrollo de Centroamérica, ésta ejerció un gran impacto a nivel internacional en orden a apoyar a la región en su andadura hacia el desarrollo. De ahí que de manera muy concreta analizara, calculara y solicitara los montos requeridos para la resurrección de una Centroamérica desvastada y anémica en su textura nacional y regional.

La Comisión recomienda por ejemplo:

- Apoyar plenamente la consolidación de la paz y la democracia conforme a las líneas del acuerdo de Esquipulas, retirando la ayuda militar externa a las fuerzas irregulares y basando sus relaciones con las naciones centroamericanas en el cumplimiento del acuerdo de Esquipulas.

- Fomentar el cumplimiento de Esquipulas II por medio de préstamos, asistencia financiera e iniciativas diplomáticas;

- No rehusar el proporcionar asistencia financiera ni ventajas comerciales a los países que cumplan el acuerdo de Esquipulas II basándose en otros criterios políticos. 


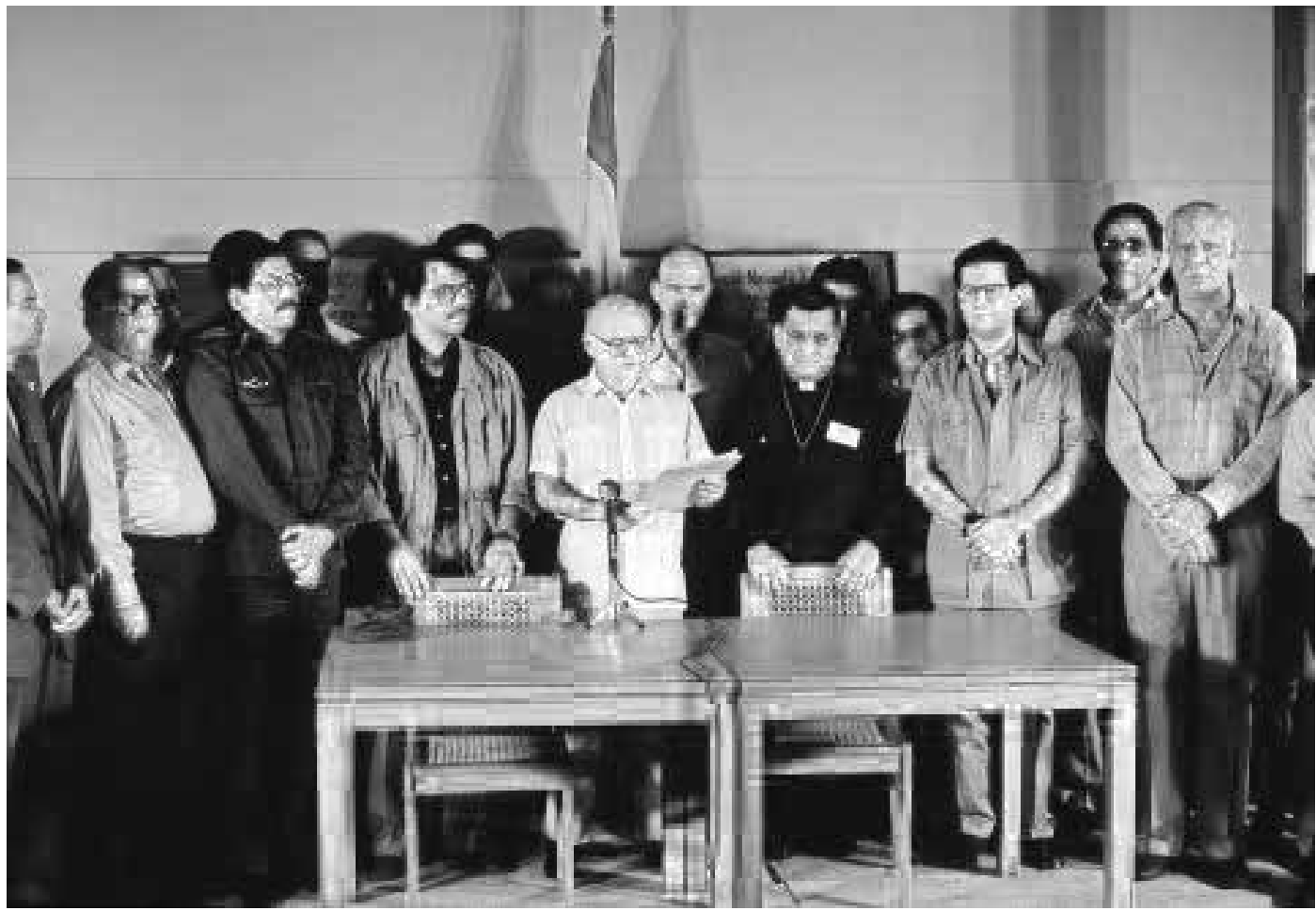

Los acuerdos de Sapoá. Foto Arturo Robles/Archivo IHNCA

Retomando el contenido, el sentido y la prospección del Informe Sandford inserto en el espíritu y material de Esquipulas II, se puede concluir que en ellos encontramos los grandes ejes de la Cultura de Paz, la que como proceso aún se sitúa lejos de la meta deseada porque todavía en Centroamérica persisten en gran parte de nuestra población elementos propios de la guerra aunque sin armas (pobreza, orfandad, migrantes, exclusión social, hambre, analfabetismo, etc.), algunas trabas contra el funcionamiento pleno de la democracia tanto participativa como representativa y muchas carencias para que la dirección y equidad del desarrollo se exprese en bien común y bienestar de toda la población.

Una Cultura de Paz sólo puede avanzar hacia su consolidación sobre los rieles de la paz duradera, la democracia auténtica y el desarrollo equitativo.

Los trazos del cuadro están muy claros, falta el artista, es decir, la voluntad política de políticos, ciudadanos y gobiernos que le den unidad y sentido a la Cultura de Paz como la realidad viva de nuestra Centroamérica.

La Cultura de Paz es una construcción personal, social, ciudadana.
Conocemos cómo se construye, podemos construirla y sin embargo parece incomodarse en nuestras manos. Está cerca pero en espera. Es contradictorio, porque por su esencia, la paz, la democracia, el desarrollo son realidades que nos son propias como personas y pueblos. La Cultura de Paz nos pertenece, aprovechemos este tesoro de vida personal y social.

\section{BIBLIOGRAFIA}

1- Acuerdo de Esquipulas II. Guatemala 07/08/1987.

2- Acuerdos de Sapóa, Nicaragua 21-23 marzo de 1988.

3- Informe Sandford. Pobreza, Conflicto y Esperanza: Un momento crítico para Centroamérica. Tecnos, 1989.

4- Acuerdos de Paz de El Salvador. Firmado en Chapultepec 16/01/1992.

5- Acuerdos de paz firme y duradera. Guatemala, 29 de diciembre de 1996.

6- Federico Mayor Zaragoza. El Derecho Humano a la Paz. UNESCO, 1997.

7- Lovo Anastasio. Cultura de Paz. Historia, Epistemología y Textos. UPOLI, Managua 2012.

25 de febrero de 2013 